\title{
N90-29627
}

\section{PERIODIC GAITS FOR THE CMU AMBLER}

\author{
Swaminathan Mahalingam \\ Graduate Student \\ Department of Mechanical Engineering \\ Carnegie Mellon University
}

\author{
Dr. Suren N. Dwivedi \\ Professor \\ Department of Mechanical and Aerospace Engineering \\ West Virginia University
}

\begin{abstract}
The configuration of the Carnegie Mellon University Ambler, a six legged autonomous walking vehicle for exploring Mars, enables the recovery of a trailing leg past the leading leg to reduce the energy expenditure in terrain interactions. In this paper gaits developed for this unprecedented configuration are described.

A stability criterion has been developed which ensures stability of the vehicle in the event of failure of any one of the supporting legs. Periodic gaits developed for the Ambler utilize the Ambler's unique abilities, and continuously satisfy the stability criterion.
\end{abstract}

\section{INTRODUCTION}

\section{THE CARNEGIE MELLON UNIVERSITY (CMU) AMBLER}

The CMU Ambler ${ }^{1}$ is being developed to study the feasibility and appropriateness of legged vehicles for rugged, barren planetary surface traversal in general and for Martian terrain in particular. It is configured to overcome the general drawbacks attributed to walking machines, such as, power inefficiency, control complexity and low payload to weight ratio. A complete description of the configuration can be found in (Bares \& Whittaker, 1988). Only features relevant to the current discussion are described in this paper.

Most walkers have been configured to have an identical number of legs attached to either side of an elongated body, similar to the arrangement in a mammal or a reptile. The configuration used by Ignatiev (Vukobratovic 1973) and that of ODEX I (Russell 1983) are exceptions, having six legs disposed symmetrically about a vertical axis.

The six legs of the Ambler too are configured to be symmetric about a vertical axis, but unlike the previous configurations there is a complete overlap of the leg workspaces. To provide this characteristic, the Ambler legs are mounted at different elevations on the central axis of the body, so that they can rotate fully around the axis (Figures $1(\mathrm{a})$ and (b)).

Each leg (Figure 2) of the Ambler has two revolute motions in the leveled, horizontal plane, in the manner of SCARA (Asada \& Youcef-Toumi, 1987, p. 8) robot arms, and a vertical telescoping link at the end of the horizontal mechanism. After each leg is positioned over the terrain with its revolute links, the vertical telescoping motion extends the foot into contact on the terrain.

1 Ambler is an acronym for Autonomous MoBiLe, Exploration Robot. 


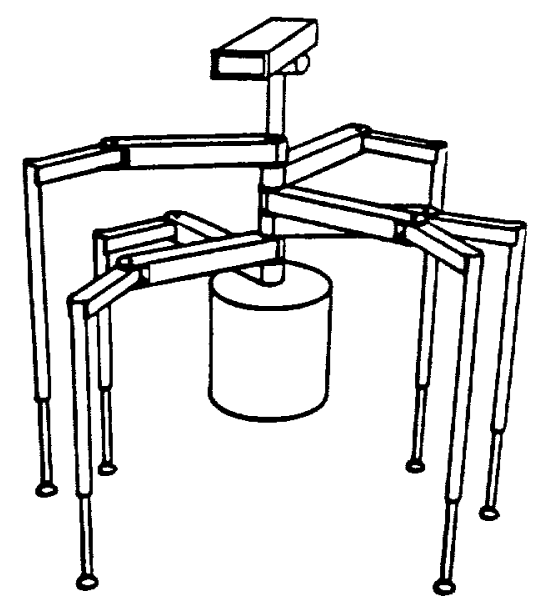

Figure 1(a). The CMU Ambler

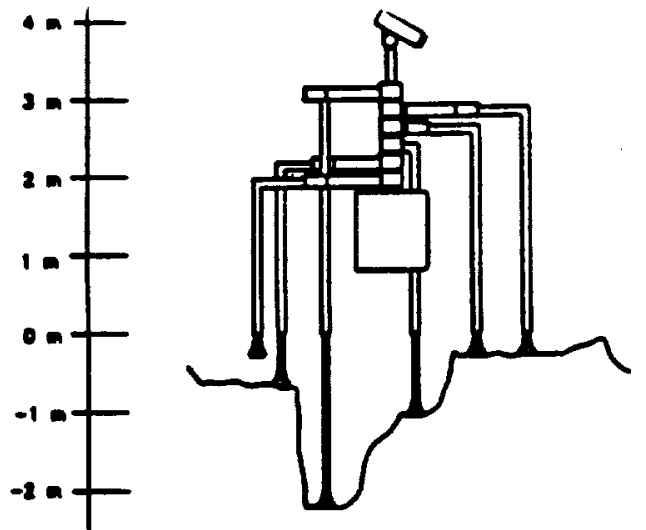

Flgure 1(b). The CMU Ambler (Side Vlew)

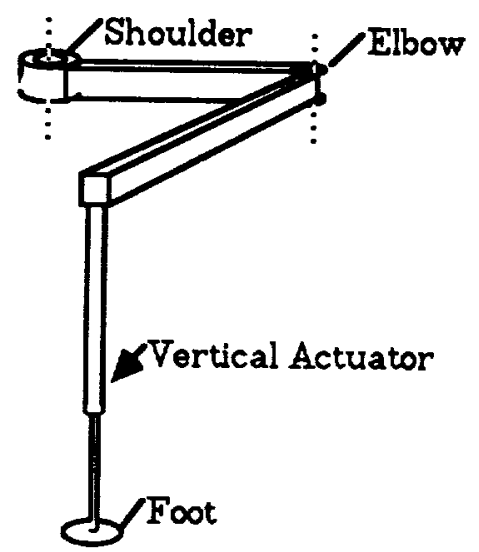

Figure 2. An Ambler Leg

The horizontal part is identical in all the legs, the vertical parts differ in height according to the leg's position in the stack - the leg which is mounted at the bottom of the stack being 
the shortest and the one mounted at the top being the tallest. The legs have a horizontal reach of two meters, the vertical actuators range from thirteen to seventeen feet.

THE NEED FOR A NEW STABILITY CRITERION FOR PERIODIC GAITS The philosophy adopted for the Ambler is that autonomous natural terrain traversal warrants the utmost conservatism to be ingrained into the walk planning. It is therefore most desirable for autonomous agents to operate, as far as is practical, in a world of facts and not assumptions.

Although not explicitly stated, an underlying assumption in the development of the previous stability criteria has been that the theoretical states of the legs (either 0 or 1) considered while quantifying stability would be physically realizable and maintainable. The philosophy adopted here is that the assumption that a footing would be secure is not enough, but that in-situ determination of the foot-terrain interaction results is necessary. It is shown in (Mahalingam, 1988) that prediction of the outcome could prove to be extremely difficult and even if possible would be of questionable accuracy. It is therefore proposed that a new stability criterion be developed for the Ambler that admits the possibility that any leg might spontaneously fail to provide support. This could occur by reason of structural or subgrade failure.

\section{THE NEED FOR NEW PERIODIC GAITS}

The most striking feature of the Ambler is that all the legs are stacked coaxially. This opens up new possibilities in walking, the most novel ones being the opportunity to recover the trailing leg past the leading leg and to pick a leg from one side and place it on the other. As this configuration is the first of its kind, gait development for it is an unexplored territory. There is a need to generate an array of periodic gaits that utilize the Ambler's unique capabilities to the fullest.

Periodic gaits are well suited for flat terrains and free gaits for very rugged conditions. But the terrain type is relative to the size and capability of the vehicle. What may be a rugged terrain for a smaller vehicle may be a flat terrain for a large vehicle. It is viewed that most of the Martian terrain will appear mild to the Ambler. There is a need to address the efficient traversal of such terrain.

\section{PERIODIC GAITS FOR THE AMBLER}

\section{THE STABILITY CRITERION}

The Ambler is expected to move with an average speed of one meter per minute and to reach that speed from rest in thirty seconds. The mass of the Ambler is expected to be about one thousand five hundred kilograms. The inertial vector, therefore, would at worst be $0.3 \%$ of its weight, excluding the resisting frictional inertia. Static stability criteria are therefore deemed to be sufficient, and the weight vector alone suffices to establish the stability criterion.

Concept of the conservative support polygon

A support pattern is a polygon comprised of the legs in the support state. The static stability criterion requires that the weight vector pass within the support pattern. While one or more legs recover, the others are expected to maintain their states to provide continued stability. If any of the support legs changes state (structural or soil failures), the number of 
points in the support pattern would be reduced, and a new support pattern would become effective which might not satisfy the static stability criterion.

It is proposed that the point of intersection of the weight vector with the support polygon be kept within a sub-region of the support pattern such that stability survives the loss of any of the supporting legs. This region is termed the conservative support polygon, and is defined below for an $\mathbf{n}$ legged machine.

The conservative support polygon

Conservative support polygon: The conservative support polygon (CSP) of an n-legged vehicle with $m$ legs on the ground is the intersection of the support patterns due to $(m-1)$ feet contacting the terrain.

The term $m-1$ represents that of the $m$ legs in the support state, one is assumed to have undergone a state change and $m$-1 legs remain in the support state. If the number of legs assumed to have undergone a state change is $n$, then $n$ cannot be greater than one, because the intersection of $m ! /((m-n) ! * n !)$ polygons for $n>1$ does not always exist. The CSP therefore safeguards against instability in the event of losing one leg only.

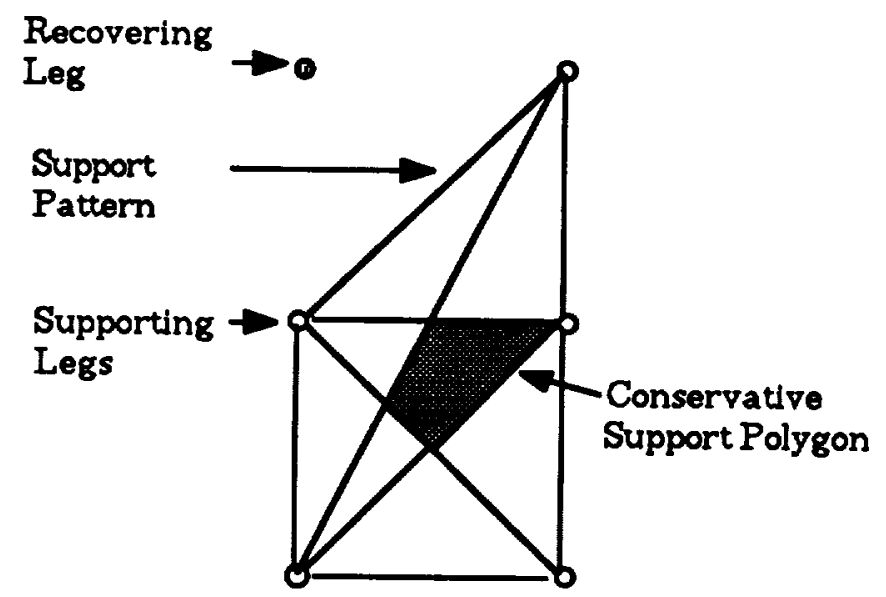

Figure 3. The Conservative Support Polygon for a slx legged walking vehicle executing crawl galt is formed by the intersection of the support patterns due to four legs in the support phase.

For the existence of the CSP, the following condition must be satisfied:

A necessary but not sufficient condition for the existence of the CSP is that the number of legs in the support phase should be equal to or greater than five, i.e., $m \geq 5$.

It is a necessary condition as the intersection of $\mathrm{m}$-1 legged support patterns do not exist for $m$ less than five and hence the CSP is undefined. It is not a sufficient condition because in some configurations, the $\mathrm{m}$ supporting legs can generate a support pattern equivalent to four or less legs.

This condition necessitates six legged vehicles to execute crawl gaits only. For the Ambler, this complements the mission's strong belief in conservatism rather than restrict the performance of the Ambler. 
Vehicles utilizing this stability criterion should ensure that the body center of gravity never lies outside the CSP. This has two implications. First, the CSP determines the allowable advancement of the vehicle before a new support pattern comes into effect. Second, gaits should provide for continuity of CSPs in consecutive support patterns.

Consecutive CSPs having point contact between them will necessitate the body center of gravity to exit one CSP and the next CSP through the contact points. The chain of consecutive CSPs therefore determines the heading of the vehicle. Consecutive CSPS having area or line contact provide more latitude for transition between CSPs than do consecutive CSPs having point contacts.

\section{Comparison with existing stability criteria}

The CSP is distinguished from other stability criteria in that the CSP does not provide a quantitative measure of stability, and therefore does not provide an optimum position to locate the body center of gravity within the CSP. The CSP establishes that as long as the weight vector intersects the support polygon within the CSP, the vehicle would be safe against instability in the event of any single leg failure. The CSP stability criterion could be expanded to include a more generalized energy stability criterion.

The CSP also supports the LSM concept. Gaits using the SM and the LSM criteria have to, depending on the terrain, dynamically determine and set appropriate limits for the stability criteria. Such techniques fail in rugged terrains where the terrain values may not attain a steady state. The CSP obviates this requirement by maintaining the body center of gravity within constant conservative LSM bounds. The LSM value is maximum when the body center of gravity is either at the lower vertex or at the upper vertex of the CSP (points $a$ and $b$ in Figure 6 ). The maximum value occurs at the mid point between the vertices. For example, in Figure 6, the CSP limits the LSM to one-fourth the distance between the leading and trailing legs.

\section{THE AMBLER PERIODIC GAITS.}

The objectives of the periodic gaits for the Ambler are to help preserve acceptable stability during locomotion, and to provide directional motion to the vehicle body with a minimum number of footfalls per distance traversed. The first objective requires that proposed periodic gaits provide conservative support polygons within consecutive support patterns having continuity to enable continuous body motion. The second criterion provides a basis of arriving at an optimal solution - a gait with optimal stability, number of footfalls per distance traversed, and directionality.

The configuration of the Ambler provides the ability to recover a trailing leg past a leading leg and the ability to pick up any leg and place it anywhere around the body Fewer footfalls per distance traversed result from the former ability, which reduces energy expenditure to the terrain as compared to conventionally configured walkers, and lessens perception and planning requirements associated with footing selection. These advantages motivate the utilization of the ability of recovering the trailing leg past the leading leg as an essential feature of the periodic gaits of the Ambler ${ }^{1}$.

1 It is to be noted that this ability renders the Ambler directional. The Ambler would be at a disadvantage to perform maneuvers which require the use of its omnidirectional capability when aligned for straight line motion. 


\section{Straight Line Locomotion}

In initial configuration, the Ambler is assumed to have three feet on either side of the body for straight line locomotion with a periodic gait ${ }^{1}$. All periodic gaits therefore initiate and complete a cycle with three feet on either side of the body. For maximum advancement of the body during straight line locomotion with periodic gaits, the configuration must be directionally biased, with maximum possible spacing between consecutive ipsilateral feet, measured along the desired direction of motion. Therefore the three ipsilateral feet are assumed to be in a straight line, parallel to and equidistant from the instantaneous longitudinal axis. To ensure uniformity in advancement throughout the cycle, the three feet are configured to have equal separation between them.

\section{The standard gait}

The Standard Gait (so called because it is expected to be the default gait of the Ambler) is a crawl gait, in which a rear leg, extended to its maximum operational length ${ }^{2}$, is recovered to the front, again extended to its maximum operational length, and the foot set on the ground. The gait assures that at this instant another leg would be ready for recovery at the rear, thereby generating a continuous motion of picking up its rear feet, one at a time, bringing it to the front, and placing it on the ground - analogous to a freestyle swimmer's hand motions.

Maximum productivity of advance argues that the foremost and the rearmost legs are to be at full extension at the same time at the initiation of each cycle, so ipsilateral feet are configured to be one-third of a cycle out of phase, and contralateral feet one-sixth of a cycle out of phase in the standard gait.

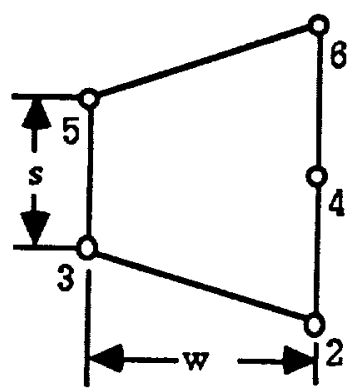

Support Pattern

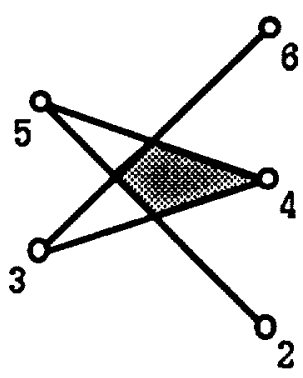

Conservative Support Polygon

Figure 4. Configuration of the Support Pattern and the CSP at the Initlation of a Cycle of the Standard Gait. Leg 1 is $\ln$ the recovery phase.

The body does not inherently have 'sides'. The desired direction of motion gives the 'heading 'of the body, which is defined at the initiation of each cycle. An instantaneous longitudinal axis of the vehicle can be defined by a line passing through the center of gravity of the body, parallel to the heading. Legs with feet on either side of this axis are referred to as being on the 'sides' of the body for convenience.

The 'orientation' of the body differs from its heading. Orientation is the rotational displacement of a body-centered co-ordinate frame with respect to an external global frame.

2 Not equal to the maximum length, which would require the two rotary links to be outstretched, introducing the possibility of control singularities. 
The support pattern of the Ambler at the initiation of a cycle of the standard gait is shown in Figure 4 together with its corresponding CSP1, illustrating the staggered placement of the feet due to the phase differences between the contralateral legs. The support patterns and the CSPs for the complete cycle are given in Figure 5.

The gait diagram in Figure 5 (a) gives information about the fraction of the cycle time a leg spends supporting the body (the duty factor, $\beta$, denoted by thick black lines) and the fraction of the cycle time a leg lags behind a reference leg in contacting the ground (the leg phase, $\left.\emptyset_{i}\right)$. The phase difference between any two adjacent ipsilateral legs $(\psi)$ is the same in the standard gait. The cycle time is divided into six equal time divisions, $t_{0}-t_{5}$ and the corresponding configurations of the Ambler are shown in Figure 5 (b). For example, at $t=$ $\mathrm{t}_{0}$, leg 1 starts recovering (denoted by a thin line in the gait diagram, and a dotted line in the configuration diagram), at $t=t_{1}$ leg 1 enters the support phase and leg 2 enters the recovery phase, and so forth. While the leg is recovering, the body is in continuous, uniform motion.

It is the objective of this gait to provide consecutive CSPs which are chained together at the vertices. The CSPs resulting from the standard gait for the Ambler are shown in Figure 5 (c). The center of gravity of the body lies at the rear end of the longitudinal diagonal of the CSP (point ' $a$ ' in Figure 6) at the initiation of a leg recovery, and lies at the front end of the same diagonal (point ' $b$ ' in Figure 6) at the time of foot set down. The two consecutive CSPs have a point contact between them, and are symmetric

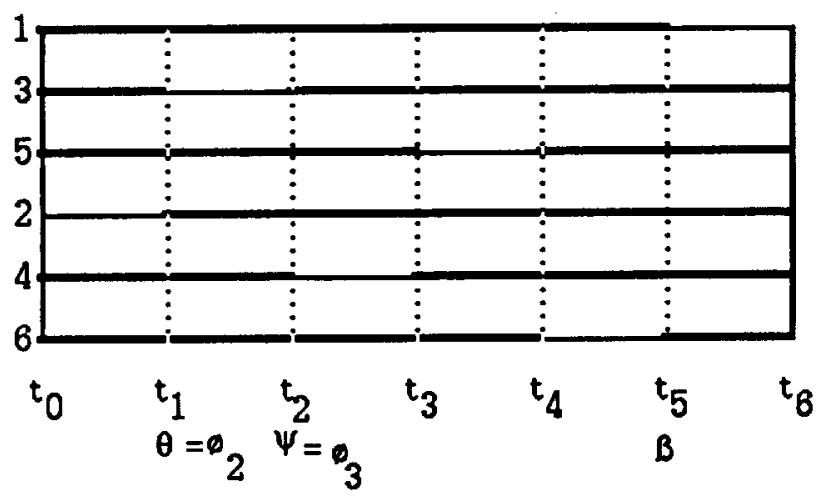

(a). Gait Diagram of the Standard Galt

1 A simple geometrical construction of the CSP for the Standard Gait is as follows: from the center foot on the side with three feet, straight lines are drawn to the two feet on the opposite side (lines 4-5 and 34 in Figure 3.7). From the two feet on the side with two feet, straight lines are drawn to the extreme feet on the other side, so that the two lines make an 'X' (lines 3-6 and 2-5). The four sided polygon obtained by the intersection of these lines defines the CSP. 


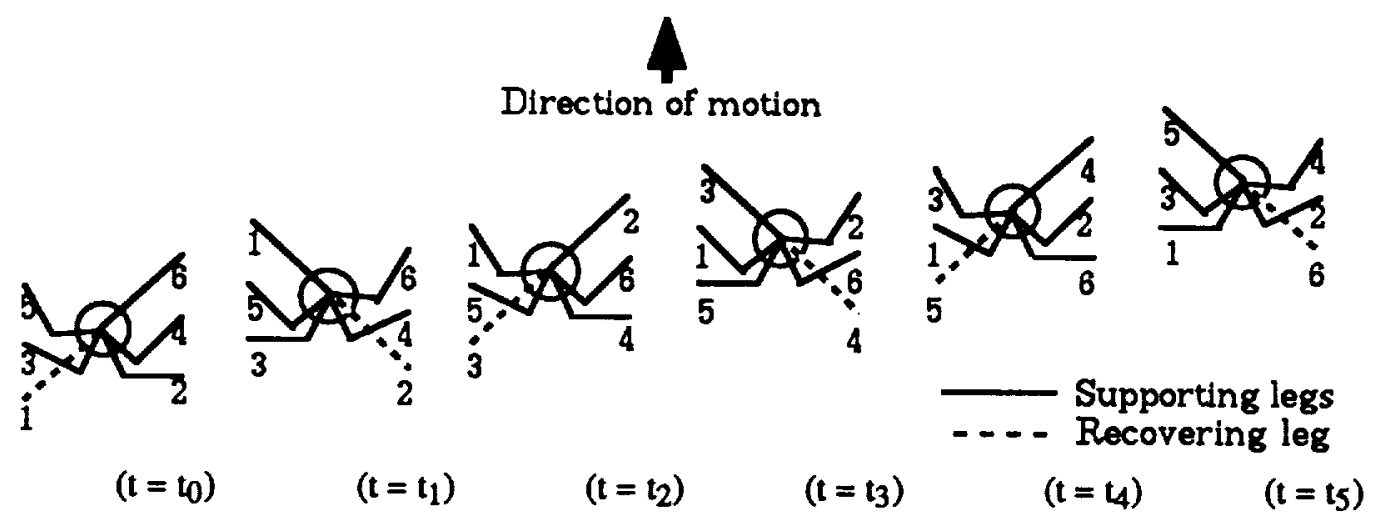

(b). Successive Configurations of the Ambler in the Standard Gait.
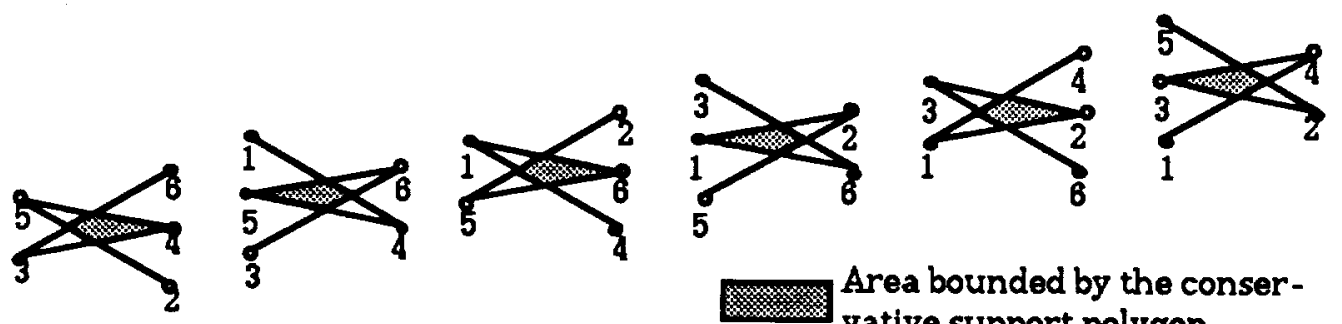

$\left(t=t_{0}\right)$

$\left(t=t_{1}\right)$

$$
\left(t=t_{2}\right)
$$

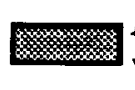

Area bounded by the conservative support polygon

$\left(t=t_{3}\right)$

$(t=4)$

$\left(t=t_{5}\right)$

(c). Successive Configurations of the CSPs in the Standard Gait.

Figure 5. The Gait Diagram, Configurations, and Conservative

Support Polygons for One Cycle of the Standard Gait.

about the contact point. Therefore, the exit point of a CSP at $t=t_{i-1}$ corresponds to an entry point of a CSP at $t=t_{i}$, thus providing continuity between consecutive CSPs. This satisfies the first requirement of the Ambler periodic gaits, making it possible for the Ambler to have continuous motion while maintaining the body center of gravity within the CSP.

Feasibility and desirability of line and area contact between consecutive CSPs

It is worth considering whether gaits exist which provide a line or area (rather than a point) contact between consecutive CSPs, and if so, whether they would provide advantages over the standard gait.

Two consecutive support patterns differ in the location of only one foot and share four feet in common. If these four feet define a parallelogram, its diagonals will divide the support plane into four quadrants. The necessary and sufficient conditions for the existence of a point contact is that

- the four common feet define a parallelogram, and

- the fifth foot of the consecutive support polygons be on the opposite quadrants defined by the diagonals of the parallelogram. 


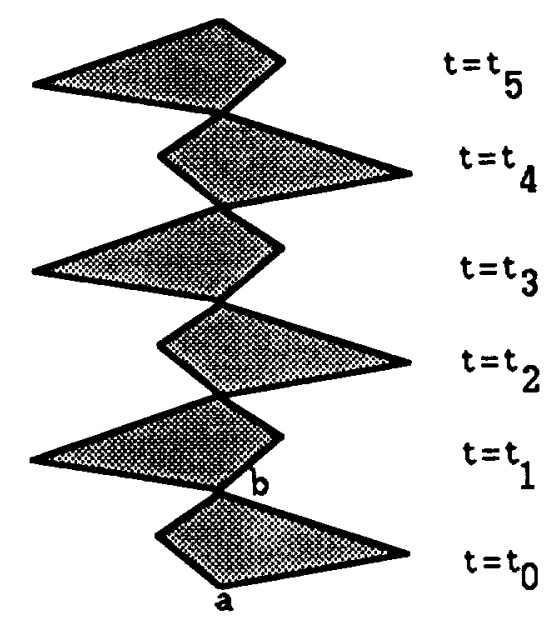

Figure 6. The Conservative Support Polygon of the Ambler with leg in recovery.

Although the generation of CSPs without point-contact is feasible, they result in gaits which have reduced average advancement per footfall. Maximum reach of the vehicle is achieved when the front and the rear legs are extended to the maximum. The maximum distance between two extreme feet in any of the gaits, which also defines the longitudinal spacing for each support pattern, is limited to twice the maximum reach of the legs. The larger the ratio between the maximum distance and the longitudinal separation, the smaller will be the advancement per footfall. Attempts to generate point-contact-free CSPs result in gaits which have larger ratios as compared to the standard gait, and therefore require a larger number of footfalls to traverse the same distance. The present view is that point contact between CSPs is not a sufficient deterrent to compromise advancement, given that the CSPs provide a high degree of guaranteed stability.

\section{Curvilinear Locomotion}

Periodic gaits which maintain the same leg sequencing as that of the standard gait, but which have different ipsilateral leg phase difference on either side, can be devised for executing motion along paths of constant curvature. Transition between two paths of different curvatures may also be accomplished by periodic gaits. Depending on the curvatures of the paths and the number of transitions from a path of one curvature to another, however, it may not be kinematically feasible to maintain periodicity continuously.

\section{Periodic Gaits with Terrain Adaptability}

The above discussed periodic gaits can be extended to have terrain adaptability features. The challenge in generating terrain adaptive gaits is to maintain $\beta$ and $\varnothing$ at their gait defined constant values while providing the freedom to select a footing from within a specified area. The parameter which lends itself to variation without affecting $\beta$ and $\varnothing$, and which is significant for defining a footing selection area is the leg recovery velocity.

By varying the velocity of the legs and keeping the time of flight constant, it is possible to recover the leg a variable distance, while maintaining the $\beta$ and $T$ values constant. Two factors limit the possible range of recovery: the control schema imposes an upper limit on 
the leg recovery velocity which defines a reachable region from the current location of the foot, and the kinematic constraints imposed on the leg due to the current configuration. Determination of this area for each foot recovery, and placement of the foot at the optimal site would result in terrain adaptation without changing the operational gait. Maintainability of the current gait for the next footfall, can therefore be assured.

\section{SUMMARY}

Terrain adaptive gaits for the CMU Ambler were presented in this paper. The conservative support polygon (CSP) was proposed as a stability criterion. The CSP ensures that the vehicle will remain stable in the event of a state change of any of the supporting legs. This requires that at least five feet remain in the support phase at any point in time.

A number of regular periodic crawl gaits were developed for the Ambler configuration, which provided continuity between consecutive CSPs. The periodic gait which provides the minimum number of footfalls per advancement is proposed as the standard Ambler gait. The standard gait parameters were determined.

\section{REFERENCES}

Asada, H. \& Youcef-Toumi, K. (1987). Direct Drive Robots: Theory and Practice. Cambridge, MA: The MIT Press.

Bares, J.E. \& Whittaker, W.L. (1988). Configuration of an Autonomous Robot for Mars Exploration. Proceedings of the SME 1988 World Conference on Robotics Research.

Mahalingam, S. (1988). Terrain Adaptive Gaits for the Ambler. MS Thesis, University of NC at Charlotte.

Messuri, D.A. \& Klein, C.A. (1985). Automatic Body Regulation for Maintaining Stability of Legged Vehicle During Rough-Terrain Locomotion. IEEE Journal of Robotics and Automation, RA-1, (3), 132-141.

Russell, M. (1983, Sep/Oct). ODEX I: The First Functionoid. Robotics Age, 12-18.

Vukobratovic, M. (1973). Legged Locomotion Robots: Mathematical Models, Control Algorithms and Realizations, 5th IFAC Symp. on Automatic Control in Space, Genoa.

\section{ACKNOWLEDGEMENTS}

This work was partially supported by the National Science Foundation under Grant No. ROA-8615072. 